preferred food that were filled in by ten children and twenty-two parents. Measurements of body composition and medical checkups were similar to those of numerous international studies of the past few years and show that a long-term change of weight is impossible to achieve by conservative means. Most of the data recorded during the 2 years remained the same and did not change. The BMI SDS in reference to age and gender did not change either, which is to be seen as a success. The active body cell mass of the children increased over the project period; as did the body fat and the extra-cellular water ratio.

Conclusions: The satisfaction of the participants with the project was very high. Twenty-five per cent of the drop outs joined a local sport club. The InForm project underlines the necessity of local multidisciplinary projects with the involvement of the families and the respective schools.

\title{
72 - e-Health tools to promote healthy eating habits
}

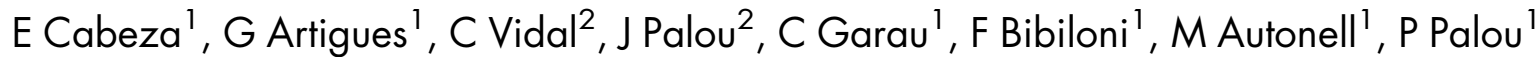 \\ and A Colom ${ }^{1}$
}

${ }^{1}$ Balearic Islands Public Health Department, Spain; ${ }^{2}$ Primary Health Care Mallorca District, Balearic Health Service, Spain

Introduction: Unhealthy diets are one of the greatest public health challenges of the 21st century. The environment of children has drastically changed in the Balearic Island during the last few decades as reflected in unhealthy dietary habits and low physical activity. Ninety per cent of the students eat breakfast regularly but only $38 \%$ of them met the nutrient recommendations.

Method: A self-administered online questionnaire has been developed in order to assess student's breakfast habits. They answer it at the school as a part of their school curricula. A personal risk score is estimated and personal recommendations are provided depending on their answers. Moreover, a overall risk profile is calculated by class and by school. Subsequently each overall school's risk is georeferenced on a map in order to help the policy makers to implement interventions addressed to these greatest risk schools.

Results: In the subsequent 4 months the online tool has assessed a total of 5429 students between the ages of 7 and 18 years old from forty-five schools. Approximately $50 \%$ of schools were classified as being at high nutritional risk.

Discussion: e-Health tools help to assess the individual risk as well as the school risk. The detection of highest risk schools might help to policy makers to plan more effective interventions to improve the healthy eating habits in these schools and the quality of the public health services and reduce health inequalities.

\section{3 - Promotion of healthy lifestyles to prevent obesity in children and adolescents in Tuscany}

\author{
G Lazzeri ${ }^{1}, V$ Pilato ${ }^{1}$, E Balocchini ${ }^{2}$, A Giannoni $^{2}$ and MV Giacchi ${ }^{1}$ \\ ${ }^{1}$ Interdepartmental Centre on Health Education and Promotion (CREPS), University of Siena, Italy; ${ }^{2}$ Tuscany \\ Region - Giunta Regionale, Direzione Generale Diritti di Cittadinanza e Coesione Sociale, Italy
}

Introduction: Recently, with the Italian Program 'Gaining Health', Tuscany has an opportunity to develop surveillance system, to lead actions and to disseminate knowledge for obesity prevention in childhood and adolescence. Objective of this presentation is to synthetically describe the systematic efforts of the Tuscany Region (Italy) in activating health promotion and disease prevention programmes at the population level. The main project developed are: 'Good practices for healthy feeding and proper physical activity in a preschool age children ( $0-5$ years)', with the strong collaboration of family paediatricians; 'National project Okkio alla salute', nutritional surveillance project with the purpose to promote the healthy growth of the primaryschool children (8/9 years); 'Health Behaviour in School-aged Children (HBSC)', with the aim to collect data on the lifestyles and the health behaviours of the 11-13-15-year-old-aged 
children in a school context; '...E vai con la frutta', good practices to promote and sustain the fresh fruit and vegetables daily consumption in schoolchildren (10-14-16 years); 'Observatory on the lifestyles', with the aim to follow-up the health promotion projects addressed to the contrast of the main risk factors for CVD; 'Ragazzinsieme', in movement between health, environment, culture and traditions (10-17 years).
Results: The overweight prevalence in childhood and adolescence in Tuscany has remained steady for 7 years.

Conclusions: The results can partially be due to the regional strategy that links the single projects in a unique program addressed to the health promotion with the involvement of all sectors.

Funding: Researches relating to this abstract were funded by Tuscany Region, 'Guadagnare Salute in Toscana'.

\title{
74 - PERISCOPE Project: how to translate healthy lifestyle in funny and enjoyable ones to preschool children and their families
}

\author{
M Caroli ${ }^{1}$, E Malecka-Tendera ${ }^{2}$ and BE Mikkelsen ${ }^{3}$ \\ ${ }^{1}$ Nutrition Unit, Department of Prevention, ASL Brindisi, Italy: ${ }^{2}$ Medical University of Silesia, Katowice, Poland: \\ ${ }^{3}$ Aalborg University, Ballerup, Denmark
}

\begin{abstract}
Aim: Aims of PERISCOPE are to increasing the discovery and knowledge of healthy never tasted foods by preschool children, training parents and teachers in eating habits modification techniques, improving children's motor abilities and physical activity levels.

Method: A two-side-book titled: 'Healthy eating the Mediterranean way: Tasty tale for children and practical tips for adults/Exercise the old fashioned way: active games for children' has been written, as health is the result of healthy eating and active lifestyle. The eating style side introduces vegetables, legumes and fruit to children through short tales. In these tales, foods and/or nutrients are positive actors of short stories to reach good health or other positive goals. A chapter with simple food health-related information, eating habits development factors and correct techniques to improve them is dedicated to parents and teachers. The
\end{abstract}

physical activity side consists of a theoretical part on motor abilities development and its relationship to psychosocial development dedicated to parents and teachers. A number of active 'old-street-games' at no cost is listed with all the instructions to perform them. The book has been used in the intervention group, during class meetings with children. Statistical analysis performed by McNemar test.

Results: The children in the intervention group ( $n$ 386) showed an increase of their fruit, vegetables and legumes intake and a higher level of physical activity as compared with the control ones ( $n 301 ; P<0 \cdot 01$ ).

Discussion: A tale book is a positive tool to improve eating habits and physical activity level in preschool children. This abstract arises from the project PERISCOPE, which has received funding from the European Union, in the framework of the Public Health Programme.

doi:10.1017/\$1368980012002492

\section{5 - Associations between stress and obesity in primary-school children}

\author{
N Michels, I Huybrechts, B Vanaelst and S De Henauw \\ 'University of Ghent, Department of Public Health, Ghent, Belgium
}

Introduction: To define correlations between stress and obesity-related factors in first to fourth grade primaryschool children.

Method: A total of 532 children (50\% male) aged 5-11 years were included from the Belgian control region of the EU 6th FP IDE-FICS cohort. BMI, body fat percentage
(BF \%) (using bioelectrical impedance, skin folds and body circumferences) and blood pressure were measured via standardized procedures. To assess stress-inducing lifeevents in the last year, the validated CLES-C questionnaire was completed during personal interview with the child. An age-adjusted score was calculated. Coping style was 